\title{
BUNDLE SHIFTS AND AHLFORS FUNCTIONS
}

\author{
M. B. ABRAHAMSE ${ }^{1}$ AND J. J. BASTIAN
}

\begin{abstract}
If $S$ is a bundle shift over $R$ with multiplicity $k$, if the boundary of $\boldsymbol{R}$ has $\boldsymbol{n}$ components, and if $\phi$ is the Ahlfors function for a point in $\boldsymbol{R}$, then $\phi(S)$ is a unilateral shift of multiplicity $k n$. It follows that a reductive algebra containing the rational algebra of a bundle shift of finite multiplicity is selfadjoint.
\end{abstract}

Let $R$ be a bounded domain in the complex plane whose boundary consists of a finite number of nonintersecting analytic Jordan curves. A bundle shift over $R$ is a pure subnormal operator with spectrum contained in the closure of $R$ and normal spectrum contained in the boundary of $R$ [2]. For a bundle shift $S$ over $R$ and for $\lambda$ in $R$, the dimension of the kernel of $(S-\lambda)^{*}$ is independent of $\lambda$ and this quantity, either a nonnegative integer or infinity, is said to be the multiplicity of $S$. The Ahlfors function for a point $t$ in $R$ is the analytic function $\phi$ which maximizes $\phi^{\prime}(t)$ within the collection of analytic functions on $R$ bounded by one and having nonnegative derivative at $t$. The function $\phi$ extends to be analytic in a neighborhood of the closure of $R$, it maps the boundary of $R$ onto the unit circle $|z|=1$, and the number of zeros of $\phi$ in $R$ (counting multiplicities) is equal to the number of components of the boundary of $R[3]$.

THEOREM. If $S$ is a bundle shift over $R$ with multiplicity $k$, if the boundary of $R$ has $n$ components, and if $\phi$ is the Ahlfors function for a point $t$ in $R$, then $\phi(S)$ is a unilateral shift with multiplicity $k n$.

Proof. It is sufficient to show that (1) the operator $\phi(S)$ is an isometry and (2) the operator $\phi(S)$ is similar to a unilateral shift of multiplicity $k n$ [2, Proposition 1.2]. If $N$ is the minimal normal extension of $S$, then $\phi(N)$ is a unitary extension of $\phi(S)$ which establishes (1). To prove (2), observe that $S$ is similar to the $k$-fold direct sum $T^{(k)}$ of the operator $T$ on $H^{2}(R)$ defined by the equation $T(f)(z)=z f(z)$ [2, Theorems 1 and 11]. Therefore, the operator $\phi(S)$ is similar to the operator $T_{\phi}^{(k)}$ where $T_{\phi}$ on $H^{2}(R)$ is defined by the equation $T_{\phi}(f)=\phi f$. The operator $T_{\phi}$ is evidently an isometry, it is pure [5, Lemma 3.8], and it has multiplicity $n$ [1, Theorem 2.14]. Thus, the operator $T_{\phi}$ is a unilateral shift of multiplicity $n$ and this establishes (2).

Received by the editors August 24, 1977.

AMS (MOS) subject classifications (1970). Primary 47B20, 47C05.

Key words and phrases. Bundle shift, Ahlfors function, subnormal operator, transitive algebra, reductive algebra, hyperinvariant subspace.

${ }^{1}$ The first author was supported in part by National Science Foundation Grant GPMPS-75-04594. 
An algebra $\mathbb{Q}$ of operators is said to be reductive if it is weakly closed, contains the identity, and if every invariant subspace for $\mathbb{Q}$ is a reducing subspace for $Q$. The algebra $Q$ is said to be transitive if it is weakly closed, contains the identity, and has no nontrivial invariant subspaces. The reductive algebra problem asks whether every reductive algebra is selfadjoint. The transitive algebra problem asks whether a transitive algebra of operators on a Hilbert space $\mathcal{H}$ must necessarily be the algebra $\mathscr{B}(\mathcal{H})$ of all operators on $\mathcal{H}$. These two problems motivate Corollaries 1 and 2 below. Corollary 3 arises from the problem of determining which operators $T$ have hyperinvariant subspaces, that is, nontrivial subspaces which are invariant for every operator which commutes with $T$.

For an operator $S$, let $\Re(S)$ denote the algebra of operators $\psi(S)$ where $\psi$ is a rational function with poles off the spectrum of $S$. Corollaries 1 and 2 follow from the theorem, the corresponding results for unilateral shifts [4], [6], [7], [8], and the fact that the Ahlfors function can be uniformly approximated by rational functions with poles off the closure of $R$. The proof of Corollary 3 is the same as that of [8, Corollary 8.19].

COROLlaRY 1. If a transitive algebra $\mathbb{Q}$ on $\mathcal{H}$ contains $\mathscr{R}(S)$ where $S$ is a bundle shift of finite multiplicity, then $\mathbb{Q}$ is $\mathscr{B}(\mathcal{H})$.

COROLlaRY 2. If a reductive algebra contains $\Re(S)$ where $S$ is a bundle shift of finite multiplicity, then the algebra is selfadjoint.

COROllary 3. For $1 \leqslant i, j \leqslant m$, let $\phi_{i j}$ be a bounded analytic function on $R$ and define $T_{i j}$ on $H^{2}(R)$ by the equation $T_{i j}(f)=\phi_{i j} f$. If the operator $T=\left[T_{i j}\right]$ on $H^{2}(R)^{(m)}$ is not a scalar multiple of the identity, then $T$ has a hyperinvariant subspace.

\section{REFERENCES}

1. M. B. Abrahamse, Toeplitz operators on multiply connected domains, Amer. J. Math. 96 (1974), 261-297.

2. M. B. Abrahamse and R. G. Douglas, A class of subnormal operators related to multiply connected domains, Advances in Math. 19 (1974), 106-148.

3. L. V. Ahlfors, Bounded analytic functions, Duke Math. J. 14 (1947), 1-11.

4. W. B. Arveson, A density theorem for operator algebras, Duke Math. J. 34 (1967), 635-647.

5. R. G. Douglas and C. Pearcy, Spectral theory for generalized Toeplitz operators, Trans. Amer. Math. Soc. 115 (1965), 433-444.

6. E. A. Nordgren, Transitive operator algebras, J. Math. Anal. Appl. 32 (1970), 639-643.

7. E. A. Nordgren and P. Rosenthal, Algebras containing unilateral shifts or finite rank operators, Duke Math. J. 40 (1973), 419-424.

8. H. Radjavi and P. Rosenthal, Invariant subspaces, Springer-Verlag, New York, 1973.

Department of Mathematics, University of Virginia, Charlottesville, Virginia 22903

Department of Mathematics, Wright State University, Dayton, Ohio 45435 (Current address of J. J. Bastian)

Current address (M. B. Abrahamse): Department of Mathematics, Loyola University of Chicago, Chicago, Illinois 60626 